\title{
Detection of Rice Plants Diseases Using Convolutional Neural Network $(\mathbf{C N N})$
}

\author{
Achmad Ramadhanna'il Rasjava ${ }^{1, *}$, Aditya Wisnugraha Sugiyarto ${ }^{2}$, Yori Kurniasari², \\ Syaifullah Yusuf Ramadhan ${ }^{3}$ \\ ${ }^{1}$ ChemistryDepartment, ${ }^{2}$ Mathematics Department, ${ }^{3}$ Statistics Department, Faculty of Mathematics and Natural Science, \\ Universitas Negeri Yogyakarta, \\ Jl. Colombo No.1, Karang Malang, Caturtunggal, Kec. Depok, Kabupaten Sleman, Daerah Istimewa Yogyakarta 55281, Indonesia. \\ Email*: ramadhanachmad44@gmail.com
}

\begin{abstract}
As a rice-producing plant, rice plant (Oryza sativa L.) is one of the most important crops in Indonesia. Rice production is increasing every year along with an increase in rice demand and population. The amount of rice production is affected by the condition of the rice plants. The worse the condition of rice plants, the rice production will also lower. Rice plant is very susceptible to diseases or pests that can reduce its productivity, including brown spot disease, leaf smut and bacterial leaf blight. As the development of science and technology, currently known as Artificial Intelligence. Artificial intelligence is a combination of several scientific disciplines such as mathematics, statistics, computer science, and even social science. Using artificial intelligence, the system now have the ability to interpret external data correctly to learn from the data and then use the learning to achieve certain goals through flexible adaptation. The artificial intelligence fields consists of several branches, such as machine learning and deep learning. Neural Network (NN) is one of the methods used in the deep learning.NN has many types, one of which is the Convolutional Neural Network (CNN). CNN is the bestknownmethod used for processingimages data compared to other types of NN. Therefore, in this study the identification of rice plants diseases was carriedout using CNN method. From this study,better results were obtained compared to other methods, obtaining 100\% accuracy for training data and $86,67 \%$ for testing data. The model obtained by the CNN method can be used for detecting 3 different types of rice plants diseases, there are brown spots, leaf smuts, or bacterial leaf blight disease based on the physical images of rice plant leaves.
\end{abstract}

Keywords: Artificial intelligience, CNN, Deep learning, Image detection, Rice plants diseases.

\section{INTRODUCTION}

Oryza sativa $\mathrm{L}$. is one of the most important staple plants in the world (Londo et al. 2006).O. sativa produces rice which is a staple food in most parts of the world, especially Indonesia (Sugiyarto et al. 2019; Kumarathilaka et al. 2018). The demand for rice is increasing every year along with the increasing population (Sakiko 2019).Nevertheless, the need for rice that continues to increase is not proportional with the increase of rice production (Adekoya and Ekeh 2019).This condition is likely caused by the rice plants condition and the growth environment that not optimal, so the rice production decreases ( $\mathrm{Li}$ et al. 2015; Asseng et al. 2017). Lower rice production by rice plants is possible due to the influence of weather, overheating air temperature, soil and air humidity, and disease (Jabran et al. 2015; Hubert et al. 2015; Kumar et al. 2009).

Observation of health and diseasesof rice plants is very important for its harvest quality and quantity, but it is very time consuming and has a lot of resources to spend (Khirade and Patil 2015), so the automatic detection of plant diseases is very necessary in the agricultural sector (Lu et al. 2017). The automatic plant diseases detection can save a lot of expenses, time, and energy. Rice plants is one of the most important staple plants need to be observed about its health and diseases.
This is due to the diseases in rice plants are able to influence the amount and quality of rice produced (Hubert et al. 2015; Kumar et al. 2009). Diseases that can attack rice plants include bacterial leaf blight (BLB) caused by the bacterium Xanthomonas oryzaepv. Oryzae. This disease can reduce rice productivity up to $40 \%$ (Sumera et al. 2017; Sumera et al. 2016; Kini et al. 2017), brown spot caused by the bacteria Helminthosporiumoryzae. This disease can reduce rice productivity by up to $30 \%$ (Tariq et al. 2012; Singh et al. 2017; Jatoi et al. 2018), and leaf smut caused by the fungus Entylomaoryzae. This disease can reduce rice productivity by $50 \%$ (Mallick et al. 2018; Prajapati et al. 2017; Mukherje et al. 2018).

In this study, we propose a method using deep learning and image processing for automatically detecting rice plants diseases using the images of its leaf. From these images, we will be able to detect rice plant diseases such as brown spot, bacterial leaf blight and leaf smut. Using this method, we are hoping that our research will be useful for agricultural sector by automatically detect rice plant diseases using its leaf images, thus saving a lot of expenses, time, efforts, and energy compared to traditional method for detecting rice plant diseases. 


\section{RESEARCH METHODS}

In this study, the general method used was presented on (Figure 1).

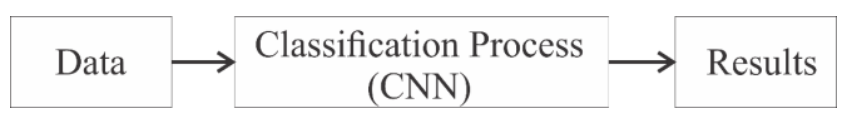

Figure 1. General research method diagram.

\section{Data}

In this study, 63 images of rice leaves was used. The data was obtained through https://archive.ics.uci.edu as 90 photographic images of rice leaves broken down to 30 diagnosed with leaf smut, 30 diagnosed with brown spot and 30 diagnosed with bacterial leaf blight.From the data, the cleaning process of data that cannot be processed is carried out so that 63 images are ready to be processed with the details of 21 photo images of leaves affected by leaf smut, 21 photo images of leaves affected by brown spots, and 21 photo images of leaves affected by bacterial leaf blight. An example of photographic data for each disease is shown in (Figure 2).

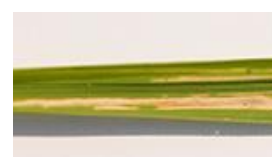

(a)

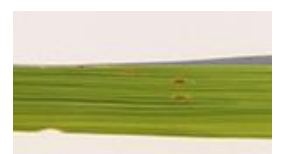

(b)

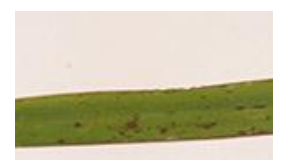

(c)
Figure 2. Image of leaves diagnosed with leaf smut (a) brown spot (b) and bacterial leaf bright (c).

\section{Classification Process (CNN)}

The next step is to carry out the learning process to get the best model in the classification of the leaf photo image. In this study the CNN method is used for classification. However, before entering into the CNN method, preprocessing is done to the leaf photo data that has been obtained, namely dividing the data into $76 \%$ for training data and $24 \%$ for testing data. Training data is used to find the best classification model based on image input data and original classification results. Then, to test whether the model is good, we use data testing. The $\mathrm{CNN}$ process is presented in (Figure 3).

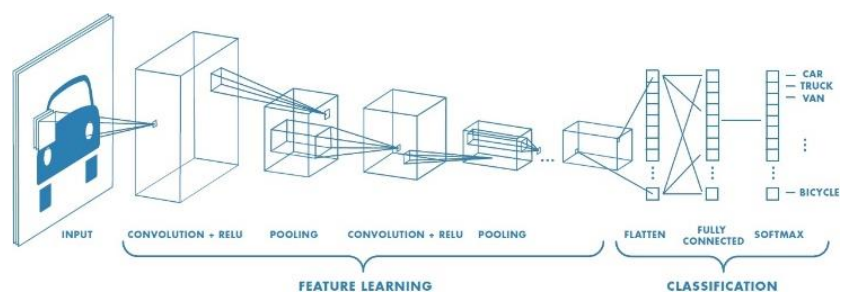

Figure 3. The Process of CNN.

\section{- Convolution Layer}

The fundamental purpose of convolution is to extract features from the input picture. Convolution uses a small square matrix, which preserves the spatial relationships among pixels, to learn image features. The convolution operations are shown in the (Figure 4). The matrix that slides the filter on the original image and performs the convolution operation is called the feature map (Zhang 2018).

INPUT IMAGE
\begin{tabular}{|r|r|r|r|r|r|}
\hline 18 & 54 & 51 & 239 & 244 & 188 \\
\hline 55 & 121 & 75 & 78 & 95 & 88 \\
\hline 35 & 24 & 204 & 113 & 109 & 221 \\
\hline 3 & 154 & 104 & 235 & 25 & 130 \\
\hline 15 & 253 & 225 & 159 & 78 & 233 \\
\hline 68 & 85 & 180 & 214 & 245 & 0 \\
\hline
\end{tabular}

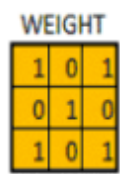

\begin{tabular}{|l|l|l|l|}
\hline 429 & 505 & 686 & 856 \\
\hline 261 & 792 & 412 & 640 \\
\hline 633 & 653 & 851 & 751 \\
\hline 608 & 913 & 713 & 657 \\
\hline
\end{tabular}

Figure 4. Convolution process with (slide = 1). (Li et al. 2008).

For every feature map, all neurons share the same weight parameter that is known as filter or kernel (yellow part in Figure 4). The filter is a feature detector for the original input picture. Different filters will produce different feature maps for the same picture. By simply adjusting the filter values, we can perform effects such as edge detection, sharpening, blurring, etc. that mean different filters detect different features, such as edges, curves, etc. from the picture.

\section{- Subsampling Layer}

The subsampling layer is also called the pooling layer. Sometimes the image is too large and we need to reduce the number of training parameters. It is required to periodically introduce a pooling layer between subsequent convolution layers. The only purpose of pooling is to reduce the size of the image space. Pooling can take many forms: Max Pooling, Average Pooling, and so on. The most common form is Max Pooling that is shown in (Figure 5).

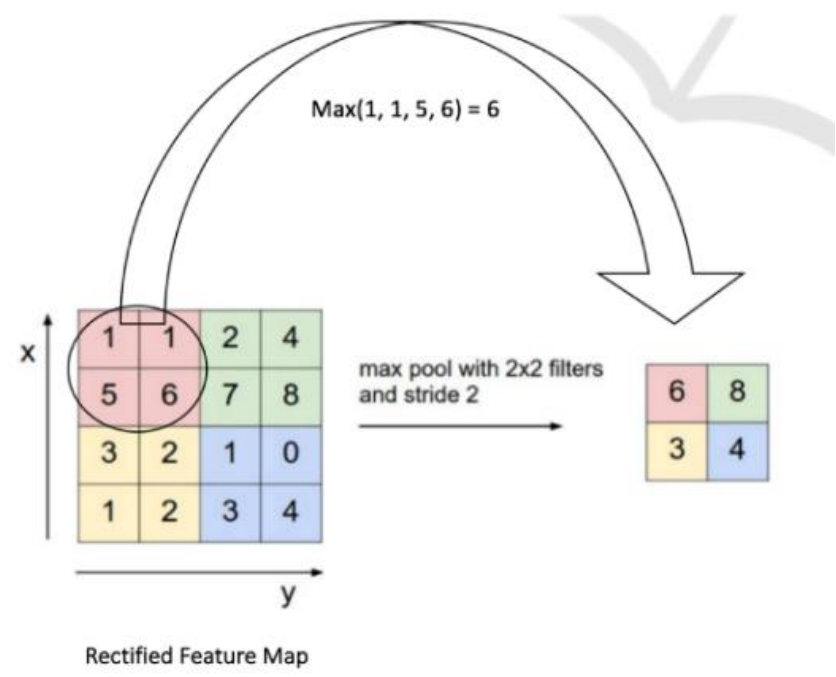

Figure 5. Max pooling process. (Prasoon et al. 2013)

Pooling operations are applied to each feature map separately. After convoluting and pooling, the image still retains most of the information as well as the size of the 
image has been reduced. The main role of the pooling layer is subsampling, which further reduces the number of parameters by removing unimportant samples in the Feature Map (Zhang 2018).

\section{- Fully Connected Layer}

Fully connected means that every neuron in the upper level is interconnected with every neuron in the next level. Convolution and pooling layer is a feature extractor, the fully connected layer is a classifier. The fully connected layer receives the output of the upper layer (which represents the feature map of the higherlevel features) and determines which category these features best fit.

For example, if the program determines the content of a picture as a dog, the feature map with higher values represent some of the more advanced features such as claws or four legs. Similarly, if the program determines the content of a picture as a bird, the feature map with higher values represent some of the more advanced features such as wings or beaks.

The fully connected layer is the Multi-Layer Perceptron that uses the softmax excitation function as the output layer. The softmax function converts a vector of any real value into a vector of elements $0-1$ and 1 . In general, a fullyconnected layer observeswhich category the advanced features most closelymatches and what weight they have.Whencalculating the weight and the dot product betweenprevious layers, we can get the correct probabilityfor the different categories (Zhang 2018).

\section{RESULTS AND DISCUSSION}

Before entering the classification process, the data augmentation stage is performed first, in which in this study the image of raw leaf photos was resized to $10 \%$ of the original size to facilitate the learning process. Because based on (Shorten and Khoshgoftaar 2019) using data augmentation the learning process will be better and have better accuracy. After that the classification process is done using the CNN method. In searching for the $\mathrm{CNN}$ architecture, trial and error techniques are used to obtain the best accuracy. In this study, an experiment was carried out by replacing many of the convolution layers of the CNN model. The results of experiments with trial and error can be seen in (Table $1)$.

Table 1. CNN Model trial results.

\begin{tabular}{ccc}
\hline Number of & \multicolumn{2}{c}{ Accuracy } \\
\cline { 2 - 3 } Layer & Training & Testing \\
\hline 1 & $93.75 \%$ & $53.33 \%$ \\
2 & $100 \%$ & $73.33 \%$ \\
3 & $100 \%$ & $66.67 \%$ \\
4 & $100 \%$ & $86.67 \%$ \\
5 & $100 \%$ & $80 \%$ \\
\hline
\end{tabular}

From the results of these experiments, that with 4 convolutional layers obtained accuracy for training data $100 \%$ and testing $86.67 \%$. The architecture of the CNN method obtained is presented in (Figure 6).

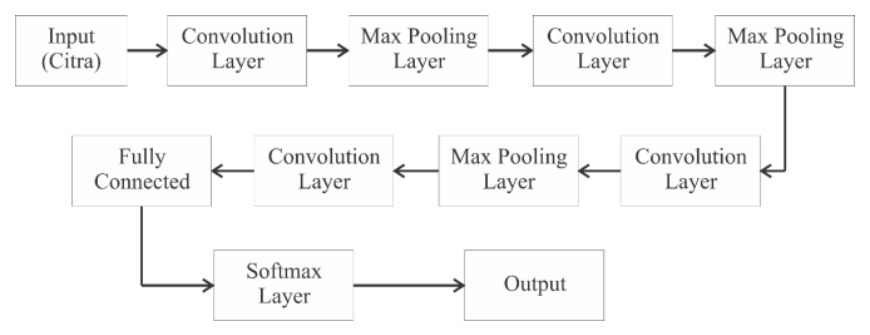

Figure 6. The most optimal CNN model architecture obtained.

The CNN model architecture consists of 4 convolution layers, 3 max pooling layers, fully connected layers and softmax layers. This means that in the model the convolution process is carried out 4 times and the re-shaping process is 3 times before entering the fully connected artificial neural network. The process of finding the model uses a maximum iteration parameter of 200. In the $\mathrm{CNN}$ model, each convolution layer contains 16 filters with a size of $3 \times 3$ and the max pooling layer has a pool size of $2 \times 2$ and a stride of 2 . The accuracy of the CNN model can be seen in (Figure 7).

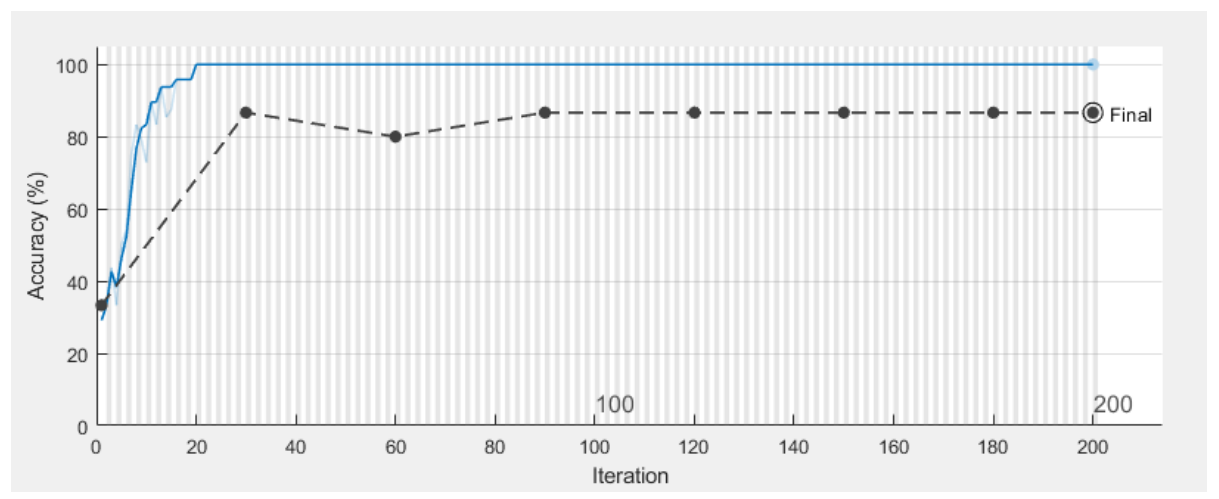

Figure 7. Accuracy results from the most optimal CNN models (straight lines show training data) and (dashed lines show testing data) 


\section{CONCLUSION}

Based on the results of the discussion, the best $\mathrm{CNN}$ model for classifying rice plant diseases based on leaf photo images is with 4 convolution layers and 3 re-shaping processes. The accuracy obtained with the model is $100 \%$ for training data and $86.67 \%$ for testing data. This result is the best compared to the accuracy for other $\mathrm{CNN}$ models so that the model can be used to classify rice plant diseases based on leaf photo images.

\section{REFERENCES}

Adekoya HO, Ekeh CM. 2019. Evaluation of Extension Agents' Communication Strategies in Promoting the New Rice for Africa Technology Among Rice Farmers in Ofada, Ogun State, Nigeria. Developing Country Studies 9(6):34-41.

Asseng S, Cammarano D, Basso B, Chung U, Alderman PD, Sonder K, Reynolds M, Lobell DB. 2017. Hot spots of wheat yield decline with rising temperatures. Glob Change Biol 23: 2464-2472.

Hubert J, Mabagala RB, Mamiro DP. 2015. Efficacy of selected plant extracts against PyriculariaGrisea, causal agent of rice blast disease. American Journal of Plant Sciences 6:602-611.

Jabran K, Ullah E, Hussain M, Farooq M, Zaman U, Yaseen M, Chauhan BS. 2015. Mulching Improves Water Productivity, Yield and Quality of Fine Rice under Water-saving Rice Production Systems. J Agro Crop Sci 201: 389-400.

Jatoi GH, Abro MA, Tariq JA, Memon S, Mangi N, Keerio AU, Gadhi MA, Hussain S,Qiu D. 2016. Screening of potential bacterial bio-control agents against the helminthosporium oryzae in-vitro conditions. Caused by brown spot of rice. Pak. J. Biotechnol. Vol. 13 (2):95-100.

Khirade SD and Patil AB. 2015. Plant Disease Detection Using Image Processing; 2015 International Conference on Computing Communication Control and Automation:768-771.

Kini K, Agnimonhan R, Afolabi O, Soglonou B, Silué D, Koebnik R. 2017. First Report of a New Bacterial Leaf Blight of Rice Caused by Pantoeaananatis and Pantoeastewartii in Togo. Plant Disease 101(1):241.

Kumar KVK, Reddy MS, Kloepper JW, Lawrence KS, Groth DE, Miller ME. 2009. Sheath Blight Disease of Rice (Oryza Sativa L.) - an Overview. BiosciBiotechnol Res Asia 6(2).

Kumarathilaka P, Bundschuh J, Seneweera S,Meharg AA. 2018. Arsenic speciation in soil-water system and their uptake by rice (Oryza sativa); Proocedings of the 7th International Congress and Exhibition on Arsenic in the Environment: 289291.

Li T, Hasegawa T, Yin XY, Zhu Y, Boote K, Adam M, Gaydon D. 2015. Uncertainties in predicting rice yield by current crop models under a wide range of climatic conditions. Global Change Biology 21:1328-1341.

Li X, Wang L, Sung E. 2008. AdaBoost with SVM-based component classifier. Engineering Applications of Artificial Intelligence 21(5): 785-795.
Londo PJ, Chiang YC, Hung KH, Chiang TY, Schaal BA. 2006. Phylogeography of Asian wild rice, Oryza rufipogon, reveals multiple independent domestications of cultivated rice, Oryza sativa. PNAS 103(25): 9578-9538.

Lu Y, Yi S, Zeng N, Liu Y, Zhang Y. 2017. Identification of rice diseases using deep convolutional neural networks. Neurocomputing 267:378-384.

Mallick DK, Ray R, Dash SR. 2018. Detection and Classification of Crop Diseases from Its Leaves Using Image Processing. Smart Intelligent Computing and Applications. Smart Innovation, Systems and Technologies 159.

Mukhereje, Maheswari NU, Basobi. 2018. Biological Control of Narrow Brown Leaf Spot and Leaf Smut Disease in Paddy Crops by Some Antagonistic Fungi. Global Journal of Medical Research, [S.1.], apr. 2018. ISSN 2249-4618.

Prajapati HB, Shah JP, Dabhi VK. 2017. Detection and classification of rice plant diseases. Intelligent Decision Technologies 11(3):357-373.

Prasoon A, Petersen K, Igel C, Lauze F, Dam E, Nielsen M. 2013. Deep feature learning for knee cartilage segmentation using a triplanar convolutional neural network; Proceeding of International Conference on Medical Image Computing and Computer-Assisted Intervention in Nagoya University, Japan, 22-26 September 2013.

Sakiko S. 2019. Consumer preference on rice as the most favorite staple food in rural Ghana; 2019 Annual Meeting Agricultural and Applied Economics Association.

Shorten C, Khoshgoftaar TM. 2019. A survey on image data augmentation for deep learning. Journal of Big Data:1-48.

Singh L, Lal LA, Kumar P, Simon S and Kumar M. 2017. Management Of Brown Spot Disease Of Rice By Safer Fungicidesand Some Bioagents. Plant Archives 17 (2):10201022 .

Sugiyarto AW, Wutsqa DU, Hendiyani N, Rasjava AR. 2019. Optimization of Genetic Algorithms on Backpropagation Neural Network to Predict National Rice Production Levels;Proocedings of the 2nd ICAITI in Bali, Indoensia.

Sumera Y, Hafeez FY, Mirza MS, Maria R, Arshad HMI, Muhammad Z, Mazhar I. 2017. Biocontrol of Bacterial Leaf Blight of Rice and Profiling of Secondary Metabolites Produced by Rhizospheric Pseudomonas aeruginosa BRp3. Frontiers in Microbiology 8:1895.

Sumera Y, Zaka A, Imran A, Zahid MA, Yousaf S, Rasul G, Arif M, Mirza MS. 2016 Plant Growth Promotion and Suppression of Bacterial Leaf Blight in Rice by Inoculated Bacteria. PLoS ONE 11(8)

Tariq JS, Ismail M, Ahmed N, Bughio HUR, Arain MA, Yasin SI. 2012.Evaluation of rice germplasm against brown spot caused by helminthosporiumoryzae in Sindh. Int. J. Agric. Appl. Sci. 4(2): 130-134.

Zhang Q. 2018. Convolutional Neural Network; Proceeding $3^{\text {rd }}$ International Conference on Electromechanical Control Technology and Transportation (ICECTT) in Chongqing, China, 19-21 January 2018. 